\title{
The Non-Uniform and Dynamic Orbits of Trans-Neptunian Objects
}

\author{
Robert B. Brown", Scott R. Dahlke \\ Department of Astronautics, United States Air Force Academy, Colorado, USA
}

Email address:

robert.brown@usafa.edu (R. B. Brown)

${ }^{*}$ Corresponding author

\section{To cite this article:}

Robert B. Brown, Scott R. Dahlke. The Non-Uniform and Dynamic Orbits of Trans-Neptunian Objects. International Journal of Astrophysics and Space Science. Vol. 6, No. 2, 2018, pp. 38-43. doi: 10.11648/j.jjass.20180601.14

Received: February 8, 2018; Accepted: March 1, 2018; Published: March 19, 2018

\begin{abstract}
Previously several authors have proposed the existence of one or more giant planets beyond Neptune to explain the non-uniform orbital elements for a dozen or fewer trans-Neptunian objects (TNOs). However, as shown here, it is not just twelve orbits that are non-randomly distributed. The distribution of the longitudes of ascending node, $\Omega$, for all of the known TNOs with perihelia beyond Neptune is also non-uniform, and this cannot be explained by observational bias. However, simulations show that $\Omega$ should become uniformly distributed within just three to five million years due to small perturbations from the known planets. Furthermore, the proposed Planet Nine cannot prevent this randomization. These results indicate it is plausible that TNOs have only been in their present orbits for a few million years or less, and there is no reason for giant, undiscovered planets to exist.
\end{abstract}

Keywords: Trans-Neptunian Objects, Kuiper Belt, Planet Nine

\section{Introduction}

Trujillo and Sheppard [1] were the first to notice that all of the dozen trans-Neptunian objects (TNOs) with semimajor axes, $a$, greater than $150 \mathrm{AU}$ and perihelia distance, $q$, greater than $30 \mathrm{AU}$ are not uniformly distributed. Surprisingly, they all have arguments of perihelion, $\omega$, close to $0^{\circ}$, meaning their perihelia lie near the ecliptic as they move from south to north. This was unexpected because perturbations from the known planets change TNOs' arguments of perihelion at different rates. As a result, after just a few million years, small perturbations should have produced a uniform distribution of $\omega$ for these dozen TNOs [2].

The clustering of $\omega$ for these twelve TNOs could not be attributed to observational bias. TNOs in elliptical orbits are brighter near perihelion, so surveys near the ecliptic are expected to find many TNOs with $\omega$ near $0^{\circ}$, meaning they are moving north as they cross the ecliptic near perihelion. However, there should be about as many equally bright TNOs discovered near perihelion moving south across the ecliptic, corresponding to $\omega$ close to $180^{\circ}$. Surprisingly, all twelve of these TNOs are moving south to north as they pass the ecliptic near perihelion, and there is no possible observational bias that could explain this. Also, the surveys that found these TNOs were often discovered away from the ecliptic, which would not have a bias for $\omega$ near $0^{\circ}$ or $180^{\circ}$. For these reasons Trujillo and Sheppard ruled out the possibility of observational bias [1].

Instead, they proposed that these orbits were created shortly after the formation of the Solar System, and $\omega$ has remained clustered for billions of years via the Lidov-Kozai effect [3] caused by an undiscovered, large planet, located $\sim 250 \mathrm{AU}$ from the Sun. They were unable, however, to explain why $\omega$ would initially be clustered near $0^{\circ}$, and not also $180^{\circ}$ as expected [4]. Another study confirmed that this clustering is not due to observational bias [5]. However, their study strongly suggested that at least two undiscovered planets are needed to keep $\omega$ from becoming randomly distributed. Later, Iorio ruled out the presence of large planets with semimajor axes between 200 and 300 AU based on the precession of $\omega$ for known planets in the Solar System [6]. This reopened the question of why TNOs with semimajor axes greater than $150 \mathrm{AU}$ and perihelia beyond Neptune's orbital distance of $30 \mathrm{AU}$ have $\omega$ grouped so closely. Why would their arguments of perihelion be clustered initially, and how 
could they remain clustered for billions of years?

More recent studies noted that the longitudes of ascending node, $\Omega$, for these TNOs with very long periods are also not uniformly distributed [7, 8]. Batygin and M. Brown studied the six most stable TNO orbits in this range and showed they all have longitudes of ascending node between $100^{\circ}$ and $126^{\circ}$ [8]. They concluded that neither the clustering of $\omega$ nor $\Omega$ is caused by observational bias. To explain how the orbital elements for these six TNOs remained similar for billions of years, they proposed that there is an undiscovered, giant planet with a semimajor axis of $\sim 700 \mathrm{AU}$ and an eccentricity, $e$, of $\sim 0.6$. They predicted this planet, which they called Planet Nine, will be discovered by 2021 [9] and provided specific locations to search for it [10]. Another study showed this hypothetical planet alone cannot explain the clustering of these six TNOs. Instead Planet Nine would cause half of these TNOs to become unstable on time scales of just dozens of Myr and eventually result in their ejection from the Solar System [11]. Therefore, that study concluded at least two undiscovered, giant planets are needed to keep these six TNO orbits clustered for billions of years. In addition, more recent discoveries of four additional long-period TNOs did not exhibit the same orbital clustering, which caused some to doubt if there is any evidence for the Planet Nine hypothesis [12].

R. Brown and Firth were the first to report a similar clustering of orbital elements for a few TNOs in the scattered disk [7]. They observed that all nine TNOs with $a>200$ AU and perihelia beyond Neptune have arguments of perihelion within $341.4^{\circ} \pm 55.5^{\circ}$ and longitudes of ascending node of $122.7^{\circ} \pm 56.6^{\circ}$. However, they noticed that there are over 1000 additional TNOs that are not randomly distributed, which led to a very different hypothesis. They discovered that neither $\omega$ nor $\Omega$ is randomly distributed for all known TNOs with $q>30 \mathrm{AU}$, even after discounting those that might be in resonance with Neptune. Instead, there are significantly more TNOs with $\omega$ near $0^{\circ}$ and $180^{\circ}$, meaning their perihelia are close to the ecliptic. They also noticed that the longitudes of ascending node are also not uniformly distributed for all known TNOs with $q>30$ AU. Instead, there are significantly more TNOs with longitudes of ascending node close to Neptune's $\Omega$ of $132^{\circ}$. This is true for TNOs with $q>30$ AU and $q>44$ AU even after eliminating TNOs that might be in resonance with Neptune. If the majority of TNOs were discovered near the ecliptic, the clustering of $\omega$ could be explained by observational bias, but is doubtful that the clustering of $\Omega$ is caused by observational bias. Given these findings, R. Brown and Firth postulated that TNOs have not been in their orbits for billions of years.

Simulations of the known TNO orbits were conducted to investigate the dynamics of their orbits, and the results are reported here. Section 2 of this paper shows that the distribution of TNOs' longitudes of ascending node are not randomly distributed today. Section 3 then describes the methods used to propagate all of these TNO orbits forward and backward in time. As shown, the distribution of $\Omega$ becomes uniform within only three to five million years due to gravitational perturbations from the known giant planets. Furthermore, perturbations from the proposed Planet Nine are unable to prevent the longitudes of ascending node from becoming uniformly distributed in this time frame. All of these results are summarized and discussed in Section 4.

\section{Distribution of $\Omega$ Today}

The arguments of perihelion, $\omega$, are not uniformly distributed today for the 1601 known TNOs with perihelia greater than 30 AU (data collected on 19 May 2016 from JPL's small body database). Instead, their values are concentrated near $0^{\circ}$ and $180^{\circ}$. This, however, is not the focus of this paper, because it could be explained by observational bias. A majority of surveys are conducted near the ecliptic, and as mentioned earlier it is much easier to discover TNOs near perihelion. Those TNOs discovered near perihelion and the ecliptic will have $\omega$ near $0^{\circ}$ if they are moving north. Assuming an even distribution of TNOs, almost as many should be discovered moving south, corresponding to $\omega$ near $180^{\circ}$, and this could explain the observed clustering of arguments of perihelion.

What is much more interesting and harder to explain is the non-random distribution of the longitudes of ascending node for all of these known TNOs. As shown in Figure 1, considerably more TNOs have $\Omega$ within $90^{\circ} \pm 70^{\circ}$, yet there are very few TNOs with $\Omega$ near $270^{\circ}$. A chi-square test easily rejected the hypothesis that this came from a uniform distribution $(\alpha=$ $0.01, p$-value $\left.<1 \times 10^{-16}\right)$. This extremely small $p$-value shows the results are very significant, and there is almost no chance that this distribution came from a uniformly distributed population.

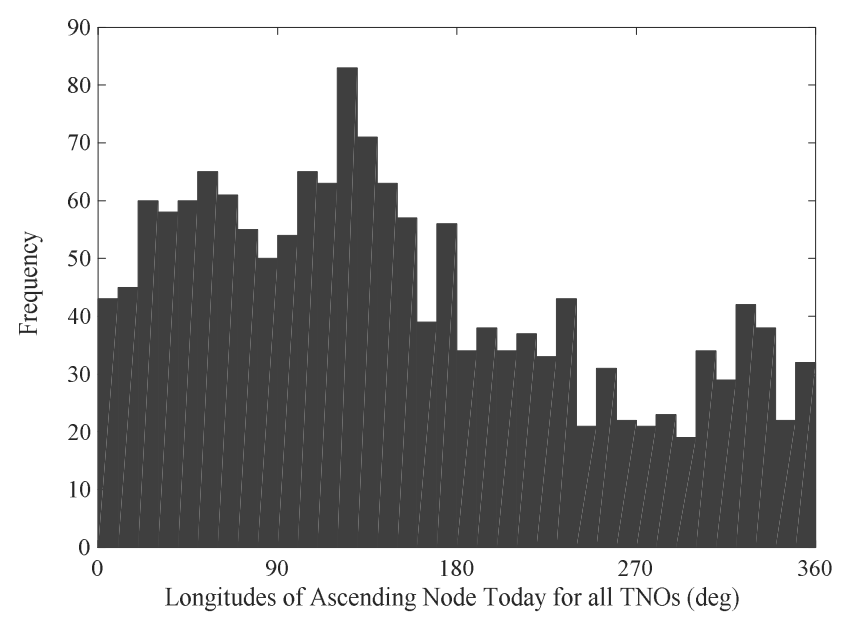

Figure 1. Distribution of the longitudes of ascending node for all 1601 known TNOs with $q>30$ AU. A chi-square test showed that $\Omega$ is clearly not randomly distributed today $\left(\alpha=0.01\right.$, p-value $\left.<1 \times 10^{-16}\right)$.

The distribution in Figure 1 was created using all of the known TNO orbits that have a perihelion beyond Neptune's orbit. However, many of these orbits lie very close to the ecliptic, and if inclination, $i$, is $0^{\circ}, \Omega$ is undefined. If $i$ is very close to $0^{\circ}, \Omega$ is almost undefined and is very sensitive to small perturbations. For these reasons, $\Omega$ is somewhat irrelevant for orbits that are close to the plane of the ecliptic. Therefore, it is reasonable to wonder if the non-random dis- 
tribution of $\Omega$ shown in Figure 1 would disappear if those TNOs with small inclinations were eliminated from this plot. To investigate this, Figure 2 shows the distribution of $\Omega$ for all TNO orbits with $q>30$ AU and $i>5^{\circ}$. There are 913 of these orbits, and their longitudes of ascending node are also not randomly distributed. As shown in Figure 2, many more orbits have $\Omega$ between roughly $20^{\circ}$ and $160^{\circ}$, while relatively few orbits have $\Omega$ around $270^{\circ}$, which is similar to the plot shown in Figure 1. This distribution was evaluated using a chi-square test, which easily rejected the hypothesis that this came from a uniform distribution $\left(\alpha=0.01, p\right.$-value $\left.=4.92 \times 10^{-8}\right)$.

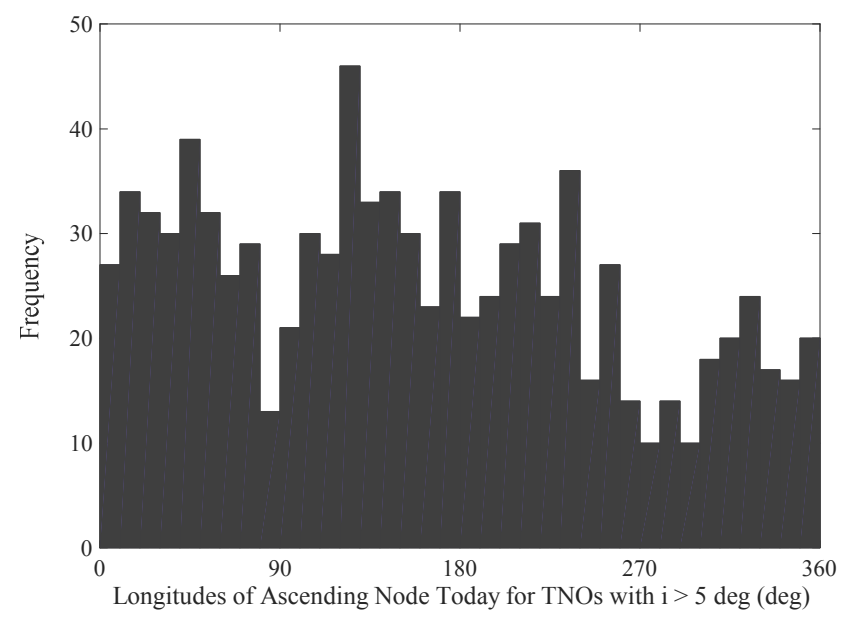

Figure 2. Distribution of the longitudes of ascending node for all 913 known TNOs with $q>30 \mathrm{AU}$ and $i>5^{\circ} \mathrm{A}$ chi-square test showed that this is also not a uniform distribution $\left(\alpha=0.01, p\right.$-value $\left.=4.92 \times 10^{-8}\right)$.

Another distribution of $\Omega$ was created for only those 660 TNOs with $q>30 \mathrm{AU}$ and inclinations greater than $10^{\circ}$. The results were very similar to Figure 2, with the same clustering of $\Omega$ between $20^{\circ}$ and $160^{\circ}$, yet relatively few TNOs have $\Omega$ near $270^{\circ}$. As before, the chi-squared test easily rejected the hypothesis that this came from a uniformly distributed population $\left(\alpha=0.01, p\right.$-value $\left.=2.84 \times 10^{-5}\right)$.

All of this indicates that the orbits of TNOs are not randomly distributed today. It is also doubtful that this is due to observational bias. As mentioned earlier, most surveys are near the ecliptic. However, these surveys would not result in the distributions shown in Figures 1 and 2 even if they only searched in one area of the celestial sphere. For example, suppose a search for TNOs looked only near the ecliptic with a right ascension of $90^{\circ}$. Those TNOs discovered moving north would have $\Omega$ close to $90^{\circ}$. Assuming TNOs were evenly distributed, there should be roughly the same number of TNOs discovered moving south, which would give them a longitude of ascending node close to $270^{\circ}$. Therefore, this hypothetical search would result in about as many TNOs with $\Omega$ close to $270^{\circ}$ as those with $\Omega$ near $90^{\circ}$, but this is clearly not what is shown in the previous figures. Although they are less common, surveys off-ecliptic are biased to discovering TNOs in the northern hemisphere, which can result in a disproportionate number of TNOs with $\omega<180^{\circ}$. However, this should not result in any bias for $\Omega$ provided these surveys are conducted over a range of right ascension angles, which is common. Therefore, it is very doubtful that observational bias can explain the clustering $\Omega$ shown in the previous figures. Batygin and Brown also ruled out observational bias when they found a similar clustering of $\Omega$ between $100^{\circ}$ and $126^{\circ}$ for six TNOs in the scattered disk [8].

A more likely explanation for the clustering of $\Omega$, is simply that the Kuiper belt is not aligned with the ecliptic. This was previously shown by Brown and Pan, who calculated that the Kuiper belt's plane has a longitude of ascending node of $81.6^{\circ}$ [13]. This is consistent with the previous figures, which show a clustering of $\Omega$ within $90^{\circ} \pm 70^{\circ}$, yet relatively few TNOs have $\Omega$ near $270^{\circ}$. However, the question remains; why is the Kuiper belt tipped? Gravitational perturbations result in nodal regression rates for each TNO that vary based on each object's inclination, eccentricity, and semimajor axis. This causes their longitudes of ascending node to change at different rates, which should quickly result in a uniform distribution of $\Omega$.

\section{Propagation of TNO orbits}

\subsection{Methods}

Previous simulations showed that small perturbations from the known giant planets cause TNOs' arguments of perihelion to change at a constant rate [1]. Because this rate of change is different for each TNO, over time the distribution of $\omega$ should become uniform unless there is an unknown force keeping their perihelia in place, such as a giant planet beyond Neptune. Jílková et al. estimated that without such a force, the arguments of perihelion for a dozen TNOs in the scattered disk would become uniformly distributed after just a few million years [2].

The same thing should happen with their longitudes of ascending node. Gravitational perturbations from the known giant planets create different nodal rates for every $\mathrm{TNO}$, which would eventually cause $\Omega$ to become uniformly distributed. The research presented here conducted simulations of all known TNOs with $q>30$ AU to analyze their dynamic stability and estimate the time required for them to develop a uniform distribution of $\Omega$.

TNO orbital elements were collected from JPL's small-body database on 19 May 2016. These were converted into position and velocity vectors, which were propagated forward and backward in time using Cowell's technique with an eighth order Runge-Kutta (RK8) integration method [14, 15]. Each simulation used a step size of ten years, accounting for the gravitational attraction from the Sun and the four known giant planets. Simulations were conducted for 1, 2, 3, 4, 5,6 , and 7 Myr both forward and backward in time.

This RK8 integrator was validated by comparing results with two other studies. Trujillo and Sheppard [1] used the Mercury integrator [16] to propagate a dozen TNOs, while accounting for perturbations from the known giant planets. Results from their Mercury integrator and the RK8 integrator were very similar. For example, simulations with the RK8 
integrator showed that it would take $520 \mathrm{Myr}$ for $2012 \mathrm{VP}_{113}$ 's argument of perihelion to increase $360^{\circ}$, which is the same length of time shown in Figure 2 of Trujillo and Sheppard's extended data [1]. For Sedna, the required time to circularize $\omega$ was calculated to be 1296.5 Myr using the RK8 integrator compared with 1300 Myr for the Mercury integrator. Results from the RK8 integrator also compared favorably with recent research by de la Fuente Marcos et al. [11]. As shown in Figure 2 of their work, when Planet Nine was included in their simulations, three TNOs became unstable after dozens of Myr and eventually were ejected from the Solar System. The RK8 integrator produced similar results for these TNOs, moving forward and backward in time when Planet Nine was included in the simulations. The close similarity of the RK8 results compared with these two other studies provided confidence that the RK8 integrator is an accurate method to propagate TNOs.

\subsection{Propagation Results}

All 1601 known TNOs with $q>30$ AU were propagated forward in time with intervals of one million years accounting for the perturbations from Jupiter, Saturn, Uranus, and Neptune. After 1 and $2 \mathrm{Myr}$, their distributions of longitudes of ascending node still did not appear to be randomly distributed $(\alpha=0.01, p$-value $<0.00001)$. However, when the TNOs were propagated 3, 4, 5, 6, and $7 \mathrm{Myr}$ into the future, there was insufficient data to reject the hypothesis that $\Omega$ would become uniformly distributed ( $\alpha=0.01, p$-values of $0.013,0.520$, $0.016,0.011$, and 0.552 respectively). As an example, the histogram of $\Omega$ after $4 \mathrm{Myr}$ is displayed in Figure 3. Notice, this plot is much more uniformly distributed than Figures 1 and 2. Additional simulations back in time indicated that 5, 6, and $7 \mathrm{Myr}$ ago, $\Omega$ would have also been uniformly distributed ( $\alpha=0.01, p$-values of $0.345,0.459$, and 0.931 respectively).

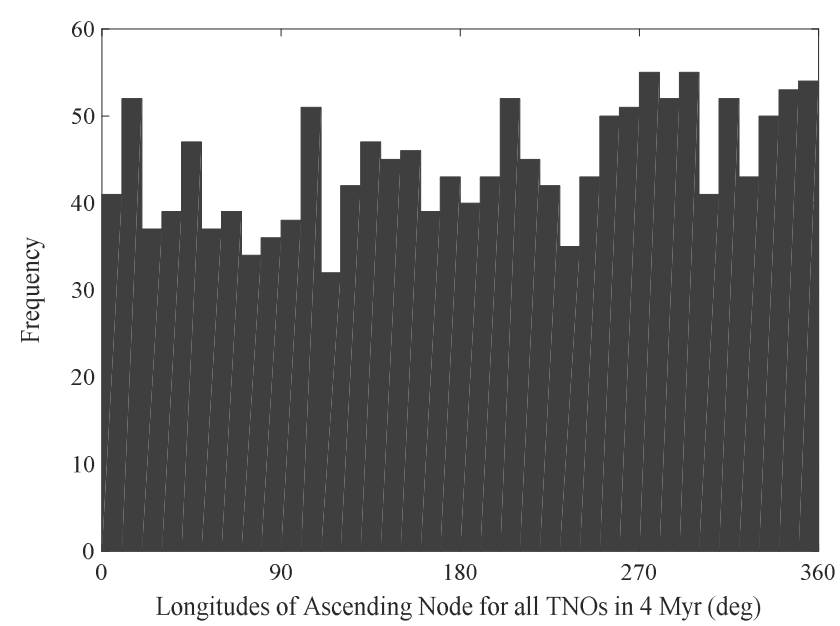

Figure 3. Distribution of the longitudes of ascending node for all 1601 known TNOs with $q>30$ AU after 4 Myr. A chi-squared test showed that this is likely from a uniform distribution $(\alpha=0.01$, p-value $=0.520)$.

Table 1 summarizes all of the statistical tests from each of the simulations forward and backward in time. The $p$-values for each chi-squared test are shown in the right column. Notice, in the middle of the table, the chi-squared test for TNOs today resulted in a $p$-value less than $1.00 \times 10^{-16}$, which indicates the distribution shown in Figure 1 is almost certainly not the result of a uniform distribution. However, for many other times, the $p$-values were greater than 0.01 (shown in bold), which means the data could not reject the hypothesis that these distributions were uniformly distributed. So, it appears that moving forward in time $3 \mathrm{Myr}$ or more, or moving backward in time at least 5 Myr, results in a uniform distribution of longitudes of ascending node. So why are their values for $\Omega$ not randomly distributed today?

Table 1. Summary of chi-squared tests to see if the distributions of $\Omega$ would be a uniform distribution after propagating all the TNOs forward or backward in time while accounting for the four known giant planets. Those p-values shown in bold could not reject this hypothesis for $\alpha=0.01$.

\begin{tabular}{ll}
\hline Propagation Time (Myr) & $\begin{array}{l}\text { p-value for hypothesis test of a uniform } \\
\text { distribution for } \boldsymbol{\Omega}\end{array}$ \\
\hline-7 & 0.931 \\
-6 & 0.459 \\
-5 & 0.345 \\
-4 & 0.0009 \\
-3 & $<0.00001$ \\
-2 & $<0.00001$ \\
-1 & $<0.00001$ \\
0 (TNOs today) & $<1.00 \times 10^{-16}$ \\
1 & $<0.00001$ \\
2 & $<0.00001$ \\
3 & 0.013 \\
4 & 0.520 \\
5 & 0.016 \\
6 & 0.011 \\
7 & 0.552 \\
\hline
\end{tabular}

\subsection{Propagation Results with the Hypothetical Planet Nine}

The gravitational attraction of a giant planet beyond Neptune would certainly affect many TNOs and might be responsible for their non-random distribution of $\Omega$ observed today. Trujillo and Sheppard proposed such a planet with a semimajor axis of $\sim 250$ AU to explain the clustering of $\omega$ for twelve TNOs in the scattered disk [1]. However, later research showed a giant planet could not exist between 200 and 300 AU based on the precession of $\omega$ for known planets in the Solar System [6]. More recently, Batygin and M. Brown proposed a giant planet with a semimajor axis of $\sim 700 \mathrm{AU}$ to explain the similar orbital elements for six of these TNOs [8]. Could this proposed planet, called Planet Nine, maintain the non-random distributions of $\Omega$ for all 1601 known TNOs with perihelia beyond $30 \mathrm{AU}$ ?

To investigate this possibility, the simulations discussed in Section 3.2 were repeated with the addition of the proposed Planet Nine. Based on previous research by other authors, Planet Nine was assumed to have a mass ten times the Earth's mass with the following orbital elements: $a=700 \mathrm{AU}, e=0.6$, $i=30^{\circ}, \omega=150^{\circ}, \Omega=113^{\circ}, v=117.8^{\circ}[8,17,18]$. The TNOs were propagated forward and backward for the same lengths of time as in Section 3.2. Occasionally, every million years, a few TNOs became hyperbolic and were ejected from the Solar System. These TNOs were eliminated from the study. For the remaining TNOs, their distributions of $\Omega$ were examined 
using a chi-square test to determine if they were uniformly distributed.

These simulations showed that the existence of Planet Nine was unable to maintain the non-random distribution for the longitudes of ascending node. After 4, 6, and $7 \mathrm{Myr}$, there was insufficient data to reject the hypothesis that $\Omega$ would be uniformly distributed ( $\alpha=0.01, p$-values of $0.017,0.088$, and 0.415 respectively). Simulations also showed that $\Omega$ would have been randomly distributed 5,6, and $7 \mathrm{Myr}$ in the past even if Planet Nine exists ( $\alpha=0.01, p$-values of $0.186,0.164$, and 0.670 respectively). These simulation results are summarized in Table 2. Similar to Table 1, the results of the statistical tests with $p$-values $>0.01$ are shown in bold, indicating the data could not reject the hypothesis that these distributions were uniform.

Table 2. Chi-squared tests to see if the distributions of $\Omega$ would be uniformly distributed after propagating all the TNOs forward or backward in time, accounting for the four known giant planets and the proposed Planet Nine. The p-values shown in bold could not reject this hypothesis for $\alpha=0.01$.

\begin{tabular}{ll}
\hline Propagation Time (Myr) & $\begin{array}{l}\boldsymbol{p} \text {-value for hypothesis test of a } \\
\text { uniform distribution for } \boldsymbol{\Omega}\end{array}$ \\
\hline-7 & 0.670 \\
-6 & 0.164 \\
-5 & 0.186 \\
-4 & $<0.00001$ \\
-3 & $<0.00001$ \\
-2 & $<0.00001$ \\
-1 & $<0.00001$ \\
0 (TNOs today) & $<1.00 \times 10^{-16}$ \\
1 & $<0.00001$ \\
2 & 0.00003 \\
3 & 0.00011 \\
4 & 0.017 \\
5 & 0.007 \\
6 & 0.088 \\
7 & 0.415 \\
\hline
\end{tabular}

These simulations demonstrate the presence of Planet Nine does not prevent the relatively quick randomization of $\Omega$ for the 1601 known TNOs with $q>30 \mathrm{AU}$. It is possible that more than one undiscovered planet is needed to maintain these non-uniform distributions for billions of years. Previous research showed that it would likely take at least two giant planets beyond Neptune to maintain the non-random orbital elements for just twelve TNOs [5] or even six TNOs [11]. Considering these studies, it would likely take numerous undiscovered planets, in very specific orbits, to maintain the orientations of 1601 TNOs. However, this would result in many other questions. It is difficult to explain how these hypothetical planets could have formed well beyond Neptune. In addition, many potential locations for giant planets have already been eliminated by observing known planets or deep space probes. For example, based on the observed orbits of known planets, one study concluded that giant planets cannot exist between 200 and $300 \mathrm{AU}$ [6]. Another study of Cassini's orbital data eliminated many other possible locations for giant planets $[17,19]$. Furthermore, a more recent study concluded that if a giant planet exists within $1000 \mathrm{AU}$ of the Sun, it would have been detected by now [20]. Given these problems, it is doubtful that there are a sufficient number of giant planets beyond Neptune to maintain the non-uniform distributions of orbital elements for 1601 TNOs.

\section{Conclusions}

A great deal of research has focused on explaining the unusual, non-random orbits of a dozen or fewer TNOs in the scattered disk. The research presented here shows that there is a more significant problem that also needs to be addressed. It is not just a few TNOs that are unusually distributed. All of the 1601 known TNOs with perihelia beyond Neptune also have non-uniform distributions of $\omega$ and $\Omega$. Although their distribution of $\omega$ could possibly be explained by the large number of surveys near the ecliptic, this could not explain their non-random distribution of longitudes of ascending node. The most plausible explanation for the clustering of $\Omega$ is the Kuiper belt is tipped relative to the ecliptic [13]. However, simulations show that within $5 \mathrm{Myr}$, either forward or backward in time, TNOs' ascending nodes should become uniformly distributed around the ecliptic due to small perturbations from the known giant planets. This indicates that either some unknown force has kept the Kuiper belt tipped relative to the ecliptic, or TNOs have been in their non-uniform orbits for only a few million years or less.

Additional simulations show that the proposed Planet Nine cannot explain the non-random distribution of $\Omega$. Instead, TNOs' longitudes of ascending node still become uniformly distributed within a few million years, whether moving forward or backward in time. It is possible that more than one giant planet beyond Neptune is responsible for maintaining the non-uniform distributions of these 1601 TNOs. However, considering previous research, this would likely require numerous giant planets $[5,11]$, and such planets could not exist between 200 and $300 \mathrm{AU}$ [6]. It is also difficult to explain how any planet formed well beyond Neptune and has remained undetected.

A simpler explanation is that TNOs, or at least a majority of them, have not been in their present orbits for more than a few million years. If so, small perturbations from the known planets would not have had sufficient time to randomize the distributions of $\Omega$. This is consistent with previous research that postulated TNOs recently migrated from inside Neptune's orbit $[7,21]$. Although this research was not able to identify a force that could have caused this migration, this TNO-migration theory does not require the existence of undiscovered, giant planets beyond Neptune.

Additional research is clearly needed. For obvious reasons the search for Planet Nine will continue. It would certainly be a remarkable discovery if Planet Nine is found, yet it would raise other questions about its origin that would need to be addressed. Other giant planets would probably also have to be discovered to explain the unusual TNO orbits presented here. However, another possibility is to conclude that TNOs have not been in their present orbits for more than about three to 
five million years. If so, there is no need to speculate that giant planets exist beyond Neptune. However, further research would be necessary to identify what forces may have recently moved TNOs into their present orbits.

\section{Acknowledgements}

This document is for information only. No U.S. Government commitment to sell, loan, lease, co-develop, or co-product defense articles or provide defense services is implied or intended.

\section{References}

[1] C. A. Trujillo and S. S. Sheppard, "A Sedna-like body with a perihelion of 80 astronomical units," Nature, Vol. 507, No. 7493, pp. 471-474 (2014).

[2] L. Jílková, S. P. Zwart, T. Pijloo, and M. Hammer, "How Sedna and family were captured in a close encounter with a solar sibling," MNRAS, Vol. 453, No. 3, pp. 3157-3162 (2015).

[3] Y. Kozai, "Secular perturbations of asteroids with high inclination and eccentricity," AJ, Vol. 67, p. 591 (1962).

[4] R. Brasser, M. J. Duncan, H. F. Levison, H. F., "Embedded star clusters and the formation of the Oort Cloud," Icarus, Vol. 184, No. 1, pp. 59-82 (2006).

[5] C. de la Fuente Marcos and R. de la Fuente Marcos, "Extreme trans-Neptunian objects and the Kozai mechanism: signaling the presence of trans-Plutonian planets," MNRAS: Letters, Vol. 443, No. 1, pp. L59-L63 (2014).

[6] L. Iorio, "Planet $X$ revamped after the discovery of the Sedna-like object 2012 VP113?" MNRAS: Letters, Vol. 444, No. 1, pp. L78-L79 (2014).

[7] R. B. Brown and J. A. Firth, "Analysis of trans-Neptunian objects and a proposed theory to explain their origin," MNRAS, Vol. 456, No. 2, pp. 1587-1594 (2015).

[8] K. Batygin, and M. E. Brown, "Evidence for a distant giant planet in the solar system," AJ, Vol. 151, No. 2, p. 22 (2016).
[9] E. Hand, "Number 9," Science, Vol. 351, No. 6271, pp. 330-333 (2016).

[10] M. E. Brown and K. Batygin, "Observational constraints on the orbit and location of Planet Nine in the outer solar system," ApJ Letters, Vol. 824, No. 2, p. L23 (2016).

[11] C. de la Fuente Marcos, R. de la Fuente Marcos, and S. J. Aarseth, "Dynamical impact of the Planet Nine scenario: N-body experiments," MNRAS: Letters, Vol. 460, No. 1, pp. L123-L127 (2016).

[12] J. Sokol, "New haul of distant worlds casts doubt on Planet Nine," Science, Vol. 356, No. 6344, p. 1221 (2017). DOI: $10.1126 /$ science.356.6344.1221

[13] M. E. Brown and M. Pan, "The plane of the Kuiper Belt," AJ, Vol. 127, No. 4, p. 2418 (2004).

[14] E. Fehlberg, "Classical Fifth-, Sixth-, Seventh- and Eighth-Order Runge-Kutta Formulas with Stepsize Control," NASA TR R-287, 52 (1968).

[15] D. A. Vallado, "Fundamentals of astrodynamics and applications (Fourth Edition)," Microcosm Press, p. 525, (2013).

[16] J. E. Chambers, "A hybrid symplectic integrator that permits close encounters between massive bodies," MNRAS, Vol. 304, No. 4, pp. 793-799 (1999).

[17] A. Fienga, J. Laskar, H. Manche, and M. Gastineau, "Constraints on the location of a possible 9th planet derived from the Cassini data," A\&A, Vol. 587, p. L8 (2016).

[18] A. Witze, "Evidence grows for giant planet on fringes of Solar System," Nature, Vol. 529, pp. 266-267 (2016).

[19] M. J. Holman and M. J. Payne, "Observational constraints on Planet Nine: Cassini Range Observations,” AJ, Vol. 152, No. 4, p. 94 (2016).

[20] A. P. Vidmachinko, "The proposed 9th planet of Solar System at a distance less than $1000 \mathrm{AU}$ is absent," International scientific conference Astronomical School of Young Scientists, May 24-25, 2017, Bila Tserkva, Ukraine, pp. 19-20 (2017).

[21] R. B. Brown, "Unusual eccentricity and inclination distributions of trans-Neptunian objects and trans-Neptunian binaries," IJASS, Vol. 6, No. 1, pp. 28-37 (2018). DOI: $10.11648 /$ j.ijass.20180601.13 